\title{
TU/e EmonOWEN

\section{Enhanced binding energy of manganese acceptors close to the GaAs(110) surface}

\section{Citation for published version (APA):}

Garleff, J. K., Wijnheijmer, A. P., Silov, A. Y., Bree, van, J., Roy, van, W., Tang, J-M., Flatté, M. E., \& Koenraad, P. M. (2010). Enhanced binding energy of manganese acceptors close to the GaAs(110) surface. Physical Review B, 82(3), 035303-1/6. [035303]. https://doi.org/10.1103/PhysRevB.82.035303

DOI:

10.1103/PhysRevB.82.035303

Document status and date:

Published: 01/01/2010

\section{Document Version:}

Publisher's PDF, also known as Version of Record (includes final page, issue and volume numbers)

\section{Please check the document version of this publication:}

- A submitted manuscript is the version of the article upon submission and before peer-review. There can be important differences between the submitted version and the official published version of record. People interested in the research are advised to contact the author for the final version of the publication, or visit the $\mathrm{DOI}$ to the publisher's website.

- The final author version and the galley proof are versions of the publication after peer review.

- The final published version features the final layout of the paper including the volume, issue and page numbers.

Link to publication

\section{General rights}

Copyright and moral rights for the publications made accessible in the public portal are retained by the authors and/or other copyright owners and it is a condition of accessing publications that users recognise and abide by the legal requirements associated with these rights.

- Users may download and print one copy of any publication from the public portal for the purpose of private study or research.

- You may not further distribute the material or use it for any profit-making activity or commercial gain

- You may freely distribute the URL identifying the publication in the public portal.

If the publication is distributed under the terms of Article 25fa of the Dutch Copyright Act, indicated by the "Taverne" license above, please follow below link for the End User Agreement:

www.tue.nl/taverne

Take down policy

If you believe that this document breaches copyright please contact us at:

openaccess@tue.nl

providing details and we will investigate your claim. 


\title{
Enhanced binding energy of manganese acceptors close to the GaAs(110) surface
}

\author{
J. K. Garleff,, ${ }^{1, *}$ A. P. Wijnheijmer, ${ }^{1}$ A. Yu. Silov, ${ }^{1}$ J. van Bree, ${ }^{1}$ W. Van Roy, ${ }^{2}$ J.-M. Tang, ${ }^{3}$ \\ M. E. Flatté, ${ }^{4}$ and P. M. Koenraad ${ }^{1}$ \\ ${ }^{1}$ COBRA Inter-University Research Institute, Department of Applied Physics, Eindhoven University of Technology, \\ P.O. Box 513, NL-5600 MB Eindhoven, The Netherlands \\ ${ }^{2}$ IMEC, Kapeldreef 75, B-3001 Leuven, Belgium \\ ${ }^{3}$ Department of Physics, University of New Hampshire, Durham, New Hampshire 03824, USA \\ ${ }^{4}$ Optical Science and Technology Center and Department of Physics and Astronomy, University of Iowa, Iowa City, Iowa 52242, USA
}

(Received 26 March 2010; published 2 July 2010)

\begin{abstract}
Scanning tunneling spectroscopy was performed at low temperature on buried manganese (Mn) acceptors below the (110) surface of gallium arsenide. The main Mn-induced features consisted of a number of $d I / d V$ peaks in the band gap of the host material. The peaks in the band gap are followed by negative differential conductivity, which can be understood in terms of an energy-filter mechanism. The spectroscopic features detected on the Mn atoms clearly depend on the depth of the addressed acceptor below the surface. Combining the depth dependence of the positions of the Mn-induced peaks and using the energy-filter model to explain the negative resistance qualitatively proves that the binding energy of the hole bound to the Mn atom increases for $\mathrm{Mn}$ acceptors closer to the surface.
\end{abstract}

DOI: 10.1103/PhysRevB.82.035303

PACS number(s): 68.37.Ef, 68.47.Fg, 73.20.Hb, 71.55.-i

\section{INTRODUCTION}

Following Moore's law of increasing computer performance, electronic semiconductor devices have been scaled down in size since the invention of the transistor. ${ }^{1}$ Nowadays the dimensions reached a level where individual dopant atoms, interfaces, and the distances in between them start to become important. ${ }^{2} \mathrm{Mn}$ acceptors in GaAs have attracted a lot of research interest especially since they have been found as a promising option to make semiconductors magnetic. ${ }^{3,4}$ Their properties in scanning tunneling microscopy (STM) measurements are also well known. ${ }^{5-7}$ The bow-tie-shaped contrast found with constant current mode STM around $+1.5 \mathrm{~V}$ is generally interpreted as the wave function of the hole bound to the Mn acceptor. ${ }^{6-8}$ Within the approach of Tersoff and Hamann, ${ }^{9}$ the STM always maps wave functions but it is not straightforward to determine whether one individual wave function is addressed that belongs to one specific Mn state. STM data measured in the constant current mode represent the integrated local density of states (LDOS) between the Fermi level $E_{F}$ and the applied voltage $\left(E_{F}+V_{\text {bias }}\right){ }^{9}$ Based on scanning tunneling spectroscopy we now show that the well-known contrast around $1.5 \mathrm{~V}$ splits into three contributions.

Strong effects of the surface have been shown: the symmetry of the Mn contrast is broken due to strain and the electric field but also due to the broken symmetry in the buckled relaxation at the $\mathrm{GaAs}(110)$ surface. ${ }^{10-13}$ Also the presence of other structures such as quantum dots ${ }^{14}$ and adsorbed atoms ${ }^{13}$ on the surface close to the $\mathrm{Mn}$ atom have been shown to disturb the electronic structure of a Mn atom. $\mathrm{Mn}$ acceptors located very close to the GaAs(110) surface show a rather intense contrast which is restricted laterally to a few lattice cells. In contrast, the wave functions of deeply embedded Mn atoms are smoothly smeared out over several nanometers. According to the basic model of a particle in a box, spatial restriction of a state is correlated with higher binding energies. Enhanced binding energy has theoretically been predicted ${ }^{15}$ for $\mathrm{Mn}$ atoms closely below GaAs(110). We studied the electronic structure of Mn acceptors located at different depths below this surface experimentally. Due to local fluctuations of the Mn concentration on the scale of typical frame sizes mapped in STM we also detected the distortion of the electronic properties of $\mathrm{Mn}$ atoms by acceptors in close vicinity.

\section{EXPERIMENTAL SETUP}

We used an Omicron low-temperature STM (LT-STM) setup operated at $5 \mathrm{~K}$ and a base pressure below $10^{-11}$ mbar. The tips were electrochemically etched from polycrystalline $\mathrm{W}$ wire, further preparation in UHV guaranteed tips of atomic resolution and stability over several hours in scanning tunneling spectroscopy (STS) mode. ${ }^{16}$ The measurements were carried out on hetero structures of Mn-doped GaAs grown by molecular beam epitaxy on $p^{+}$-doped substrates in a cross-sectional geometry (X-STM). The exact structure of the samples as well as the procedure applied to approach the heterostructures are described in Ref. 13.

On the cleaved surface we performed constant current topography in order to find a suitable area that contains Mn atoms buried in different depths below the surface and that is free of step edges and other unwanted defects, which complicate the interpretation of the data. On ensembles of Mn acceptors residing at different depths below the surface, as shown in Fig. 1, $I(V)$ spectra were acquired at every pixel of the topographic image [current imaging tunneling spectroscopy (CITS), see e.g., Ref. 17]. The depth of individual Mn acceptors below the $\operatorname{GaAs}(110)$ surface is obtained from the STM topographies. ${ }^{13}$ Figuring out the absolute depth can be difficult for $\mathrm{Mn}$ acceptors in deeper layers but the relative depth of the acceptors imaged in one frame can be identified unambiguously. The $\mathrm{Mn}$ atoms will be referred to as $\mathrm{Mn}_{2}$ to $\mathrm{Mn}_{11}$, with the depth in atomic layers as an index. Focusing on the ensemble of $\mathrm{Mn}$ atoms we adjusted the stabilization voltage $\left(V_{\text {bias }}\right)$ applied to the sample and the setpoint current in order to minimize the topographic contrast of the embed- 


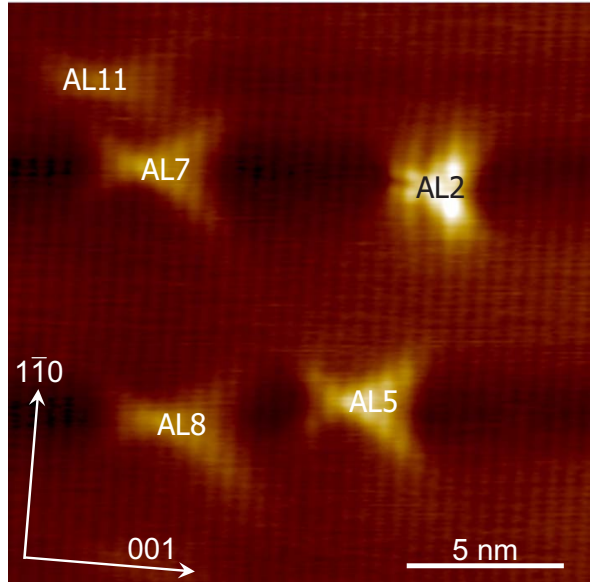

FIG. 1. (Color online) STM topography image of an ensemble of $\mathrm{Mn}$ acceptors in GaAs(110) measured at $+2 \mathrm{~V}$ and $30 \mathrm{pA}$. The depth of the acceptors below the surface (Ref. 13) is given in the image (in units of atomic layers counting the surface layer as 1).

ded $\mathrm{Mn}$ atoms. Possible artifacts in the tunneling spectra resulting from a changing tip-sample distance are avoided in this manner. Typical parameters are $V_{\text {bias }} \approx+2.3 \mathrm{~V}$ or $V_{\text {bias }} \approx-1.5 \mathrm{~V}$ with a feedback current of up to $3 \mathrm{nA}$. At every pixel on a grid of up to $256^{2}$ we performed $I(V)$ spectroscopy measurements. Each $I(V)$ curve consisted of typical 330 voltage steps between $-2 \mathrm{~V}$ and $+2 \mathrm{~V}$. After smoothing the spectra with Gauss filters and cubic spline fits, and numerical differentiation, the spectroscopic resolution was $\sim 50 \mathrm{mV}$ on the external voltage scale. In the resulting data matrix $d I / d V(x, y, V)_{z_{0}}$ we have the full information to study lateral properties of the LDOS as a function of the energy.

\section{EXPERIMENTAL RESULTS}

Within the spectroscopic information we focus on the voltage range where the Mn-induced peaks appear. In the following we will discuss the three main aspects observed around the Mn atoms in the $d I / d V$ maps shown in Fig. 2. First of all, the CITS maps show the characteristic bow-tieshaped contrast which is well known from constant current topography images of buried $\mathrm{Mn}$ atoms in $\mathrm{GaAs}$ at positive polarity around $+1.5 \mathrm{~V} .6,8,12,13$ The actual spectroscopic position depends on the depth of the addressed $\mathrm{Mn}$ atom below the surface as is shown in Fig. 2. The Mn atoms deep below the surface show the bow tie already around $+0.8 \mathrm{~V}$, see Figs. 2(a) and 2(b) whereas the voltage has to be increased to $+1.5 \mathrm{~V}$ in order to find the contrast stemming from $\mathrm{Mn}_{2}$ much closer to the surface, see Figs. 2(e) and 2(f). Second, the Mn state consists of three peaks as will be discussed later. However, the CITS data show that all of them are characterized by a very similar bow-tie-shaped pattern, and cannot be decomposed into different lateral contributions appearing at different sample voltages. The third observation concerns the LDOS at voltages slightly below the voltage at which the Mn-induced contrast in the band gap is addressed. At $\sim 1 \mathrm{~V}$ [see Fig. 2(b)] large blurry rectangular features are observed around the Mn atoms. With increasing voltage the lateral

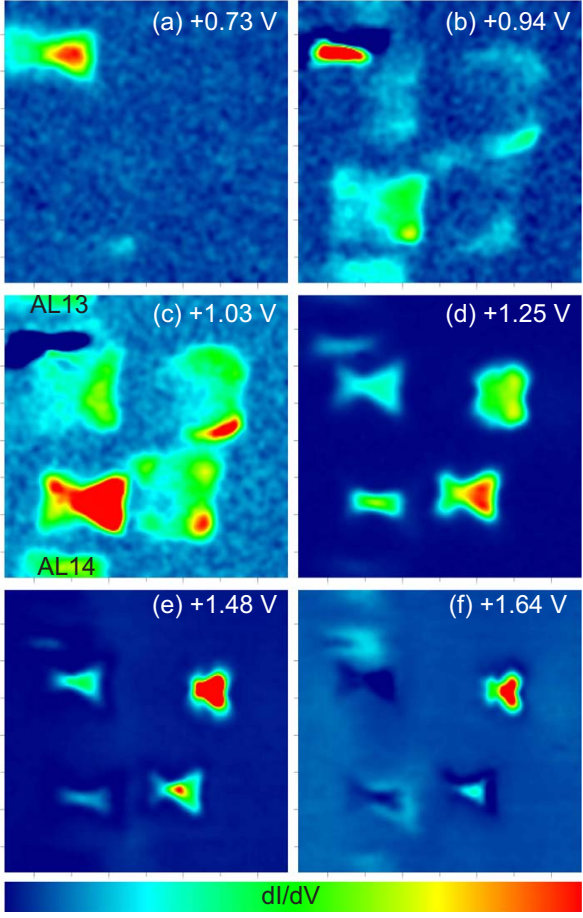

FIG. 2. (Color online) $d I / d V$ maps of the Mn ensemble shown in Fig. 1. The voltage is indicated in each image.

extension of the rectangles shrinks whereas their contrast amplitude increases, see Figs. 2(c) and 2(d). In this process, the rectangular shape deforms, and the depth-specific $\mathrm{Mn}$ induced patterns evolve; bow ties for $\mathrm{Mn}$ in deeper layers and more asymmetric shapes for Mn close to the surface, ${ }^{13}$ see Figs. 2(c)-2(f). The decreasing extension of the rimlike rectangular feature with increasing voltage shows the same voltage dependence as the rings of ionization for Mn acceptors in InAs. ${ }^{18}$

The decreasing diameter of the rings surrounding Mn acceptors in InAs with increasing voltage was explained by the tip-induced band bending (TIBB) (Ref. 19) that decreases with increasing distance from the tip and with decreasing voltage. The distance-dependent nature of this electrostatic effect results in the circular symmetry of the rings. The noncircular symmetry of the rectangular rim surrounding the $\mathrm{Mn}$ atoms in $\mathrm{GaAs}$ proves that mapping the $\mathrm{Mn}$ wave function is fully entangled with the mechanism behind this feature and that a description purely based on electrostatics is not sufficient. A second deviation of the observed rectangles from the rings of ionization can be seen where the rims of neighboring $\mathrm{Mn}$ atoms overlap. In that case the size of the rim around $\mathrm{Mn}$ atoms in GaAs does not significantly change whereas the rings of ionization around $\mathrm{Si}$ in $\mathrm{GaAs}$ and $\mathrm{Mn}$ in InAs are reduced in diameter where neighboring rings overlap. ${ }^{18,20} \mathrm{We}$ conclude that the Coulomb effect leading to a ring of ionization and mapping genuine properties of the Mn wave function are strongly entangled resulting in the rectangular rims around $\mathrm{Mn}$ atoms below $\mathrm{GaAs}(110)$.

The remainder of the paper will focus on the qualitative and quantitative interpretation of the voltages needed to address Mn atoms that clearly depend on the depth of the addressed Mn atom below the surface. Finally we will address 


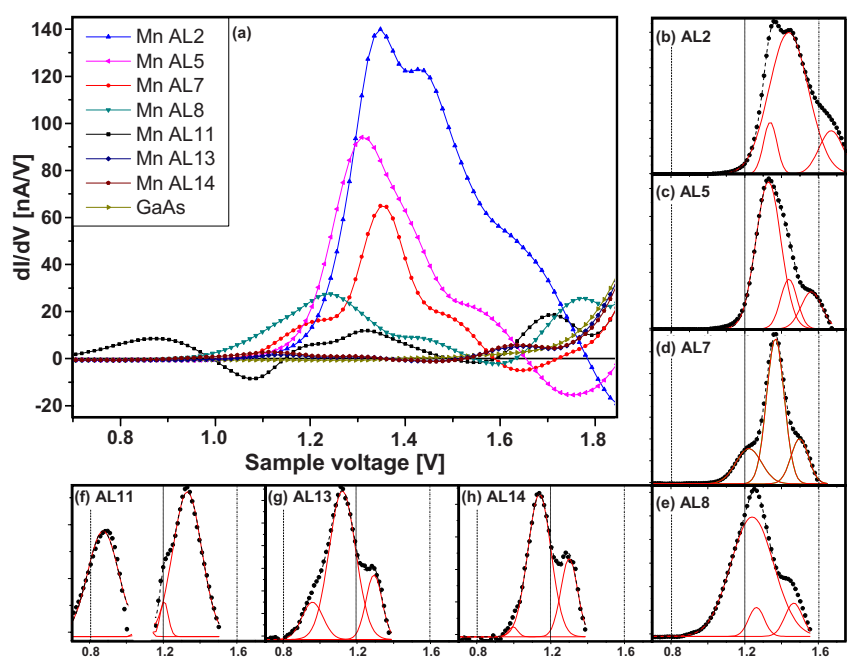

FIG. 3. (Color online) (a) Differential conductivity spectra on $\mathrm{Mn}$ acceptors in different depths below GaAs(110). (b)-(h) Gaussian fits to the Mn-induced peaks in the gap: black dots=raw data, red full lines=individual Gaussians, black dashed line=sum of the Gaussians; depth of the Mn atom as indicated.

the threefold splitting of the Mn acceptor peak.

Laterally averaging over the spectra on top of individual Mn acceptors in different atomic layers is used to study the depth dependence of the spectroscopic signature of the $\mathrm{Mn}$ acceptors. Around each of the five specific Mn atoms identified in Fig. 1, the spectra were averaged in a box of $\sim 2 \times 2.5 \mathrm{~nm}^{2}$ ( $\sim 900$ individual spectra). The resulting averaged spectra are plotted in Fig. 3(a). We compare the spectra taken on the five acceptors with an average spectrum on the bare GaAs surface in the same data set as a reference. In the upper left and lower left corner of Fig. 2(c) two additional $\mathrm{Mn}$ atoms are buried deeper below the surface than 11 atomic layers (AL). We include them in the further discussion. Based on the relative strength of their contrasts, their depth is roughly estimated to be $13 \mathrm{AL}$ and $14 \mathrm{AL}$ below the surface.

The main spectroscopic signature of the Mn acceptors is found in the band gap below the onset of the conduction band of GaAs, which is in good agreement with earlier publications for spectroscopy on $\mathrm{Mn}$ in InAs, ${ }^{21}$ and for $\mathrm{Mn}$ in the first layer of GaAs. ${ }^{7}$ It also fits to the reported position of the Mn-induced contrast in constant current topographies on Mn-doped GaAs. ${ }^{6,8,12,13}$ Figure 3(a) highlights this voltage range. The Mn signature consists of three peaks that are followed by negative differential conductivity (NDC)..$^{22,23}$

The Mn-induced peaks are quantitatively characterized by Gaussian fits. The fits to the peaks in the band gap are shown in the insets in Fig. 3. Correct assignment of the first, second, and third peak is crucial because comparing wrong peaks with each other would severely distort the derived depth dependence of the peak positions. The spectroscopic position of the NDC was used as a landmark in order to identify the corresponding peaks for the $\mathrm{Mn}$ atoms in different depth. The resulting peak positions from the Gauss fits are summarized in Table I and plotted versus the depth of the corresponding Mn atom in Fig. 4. This quantitatively confirms the trend that the peaks in the band-gap shift to lower voltages
TABLE I. Results of fitting three Gaussians to the $d I / d V$ spectra measured at the Mn atoms in different atomic layers. * peak 1 of $\mathrm{Mn}_{11}$ is shifted due to other $\mathrm{Mn}$ atoms close by.

\begin{tabular}{lllllllr}
\hline \hline & $\mathrm{Mn}_{2}$ & $\mathrm{Mn}_{5}$ & $\mathrm{Mn}_{7}$ & $\mathrm{Mn}_{8}$ & $\mathrm{Mn}_{11}$ & $\mathrm{Mn}_{13}$ & $\mathrm{Mn}_{14}$ \\
\hline Peak 1 (mV) & 1338 & 1329 & 1222 & 1227 & $874^{*}$ & 996 & 959 \\
Peak 2 (mV) & 1439 & 1440 & 1367 & 1248 & 1207 & 1139 & 1121 \\
Peak 3 (mV) & 1669 & 1562 & 1498 & 1449 & 1328 & 1300 & 1293 \\
\hline \hline
\end{tabular}

for acceptors deeper below the surface. The uncertainty of the resulting peak positions is $\sim 5 \mathrm{mV}$. The amplitude and width of the peaks, however, do not show unambiguous trends. In general, features stemming from acceptors close to the surface are stronger than those induced by deeply buried $\mathrm{Mn}$ atoms. In most cases the second peak is the strongest of the threefold peaks in the band gap.

A closer look at the spectroscopic signature of $\mathrm{Mn}_{11}$ in Fig. 3, Table I, and Fig. 4 reveals that the lowest energy peak of this $\mathrm{Mn}$ atom appears at significantly lower voltage than expected from a linear fit. The peak stemming from $\mathrm{Mn}_{11}$ lies even below the corresponding peak belonging to $\mathrm{Mn}_{13}$ and $\mathrm{Mn}_{14}$. Figures 1 and 2(c) show that $\mathrm{Mn}_{11}$ is located close to $\mathrm{Mn}_{7}$ and to $\mathrm{Mn}_{13}$. The lateral distance to both neighbors is around $\sim 2.7 \mathrm{~nm}$. Peaks at reduced voltages are systematically found for $\mathrm{Mn}$ atoms situated in close vicinity to neighboring acceptors in all our measurements. This supports the conclusion that the interactions between neighboring $\mathrm{Mn}$ atoms lower the spectroscopic position of the first peak in the band gap, consistent with Ref. 7. The critical interaction length of $\sim 3 \mathrm{~nm}$ is much larger than the average distance between the defects at the metal-insulator transition. ${ }^{24} \mathrm{Mn}$ acceptors deeper than $\sim 2.5 \mathrm{~nm}$ below the surface are hardly visible in topographic images; this depth is very similar to the critical interaction length. Therefore the local neighborhood of the individual $\mathrm{Mn}$ atoms results in shifted peak positions similar to the variations between individual emitters resulting in inhomogeneous broadening in photo luminescence experiments.

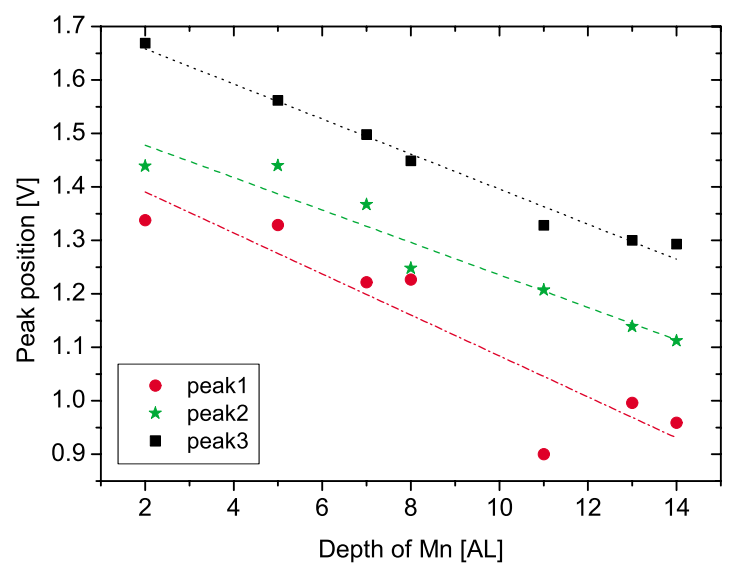

FIG. 4. (Color online) Peak positions of the Mn-induced features in the band gap for $\mathrm{Mn}$ atoms in different depth from the Gaussian fits shown in Fig. 3. The symbols red dots, green stars, and black squares depict the peaks 1, 2, and 3 in the gap. 

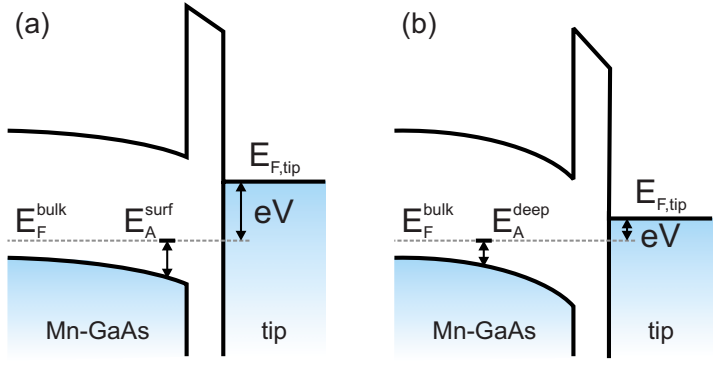

FIG. 5. (Color online) Schematic band bending of the $\mathrm{Mn}: \operatorname{GaAs}(110)$ surface in case of lining up a Mn atom (a) close to or (b) deep below the surface with $E_{F}^{\text {bulk }}$.

Splitting of the acceptor levels due to interaction with neighboring $\mathrm{Mn}$ atoms has been predicted theoretically for bulk Mn-GaAs. ${ }^{25}$ The reduced energetic position of the lowest state depends on the relative position of both Mn atoms. Our observation of a peak at significantly lower voltage for $\mathrm{Mn}$ atoms located close to each other confirms the theoretical prediction qualitatively. The limited visibility of $\mathrm{Mn}$ atoms in deeper layers, and their specific properties close to the surface hinder a quantitative comparison with Ref. 25. This splitting was measured experimentally between atoms in the surface layer ${ }^{7}$ but until now had not been demonstrated for subsurface magnetic dopant pairs.

\section{ENHANCED BINDING ENERGY}

It is tempting to interpret the shifted spectroscopic position of the Mn-induced peaks in the band gap directly as an enhanced binding energy for Mn atoms closer to the surface. A similar argument was used for $\mathrm{Mn}$ atoms in the first atomic layer. ${ }^{7,15}$ However, the situation is more complex since the Fermi level $E_{F}$ is not pinned on GaAs(110), and TIBB (Ref. 19) cannot be neglected. Quantitative calculations of the TIBB are challenging because several crucial parameters are only known by estimation, e.g., geometry and work function of the tip, and distance between tip and sample. We therefore draw qualitative conclusions on the effect of the depth dependence of the Mn-induced features before we discuss the estimated binding energy of Mn atoms in different layers below the surface.

\section{A. Qualitative analysis}

Addressing $\mathrm{Mn}$ atoms closer to the surface at increased voltage rises the question on the mechanism behind the Mninduced peaks. In general, a peak in $d I / d V$ stems from an additional tunneling channel. In the case of $\mathrm{Mn}$ in GaAs, this happens when the addressed acceptor state close to the surface $\left(E_{A}^{\text {surf }}\right)$ lines up with the Fermi level deep in the sample, $E_{F}^{b u l k}$, as schematically depicted in Fig. 5. A second ingredient for interpretation is the negative differential conductance following the Mn-induced peaks in the band gap. NDC can be explained in a straightforward manner by the energy-filter mechanism which is briefly introduced here. The tunneling current flows from the tip first into the empty acceptor state of the addressed $\mathrm{Mn}$ atom close to the surface. Then in a second step, the electrons have to reach an empty state in the partially filled acceptor band in the bulk at the same energy. The decaying TIBB toward the bulk allows to align $E_{A}^{\text {surf }}$ with $E_{F}^{\text {bulk }}$. Increasing the external voltage lifts $E_{A}^{\text {surf }}$ above $E_{F}^{b u l k}$, and the tunneling channel through the $\mathrm{Mn}$ state close to the surface is closed because the electrons can no longer be drained into the impurity band in the bulk. Blocking the tunneling channel thus decreases the current for increased voltage and explains the observed NDC. Details can be found in Ref. 23.

Assuming the binding energy of the acceptor to remain unchanged close to the surface, $E_{A}^{\text {surf }}=E_{A}^{\text {bulk }}$, means that it lines up with $E_{F}^{b u l k}$ when the bands are flat. In this situation, the TIBB and its decay toward the bulk both vanish. Therefore all $\mathrm{Mn}$ atoms in different depths would cross $E_{F}^{\text {bulk }}$ at exactly the same external voltage, and no depth dependence would be observed for the Mn-induced peaks. This is clearly not the case in our experiments as shown in Fig. 4. We conclude that $E_{A}^{\text {surf }}$ indeed depends on the depth of the Mn atom below the surface. In case of upward (downward) TIBB needed to achieve $E_{A}^{\text {surf }}=E_{A}^{b u l k}$, the Mn binding energy will be decreased (increased) close to the surface.

We measured the flat-band voltage independently by $z\left(I_{T}\right)$ (Ref. 23) spectroscopy, resulting in a flat band voltage of $2.5 \pm 0.5 \mathrm{~V}$, clearly above the Mn-induced peaks. In agreement with Ref. 22 the Mn-induced peaks and the NDC are detected at downward TIBB, proving the enhanced binding energy of Mn atoms close to the surface. Figures 5(a) and 5(b) qualitatively compare the band bending needed to address an acceptor close to the surface [5(a)] and deeper in the crystal $[5(b)]$. The higher voltage needed to align the acceptor close to the surface causes a smaller total TIBB than the lower voltage at which the deeper acceptor aligns with the impurity band. Due to the rapid decay of the TIBB toward the bulk, the local TIBB at the acceptor site is still higher for the acceptor close to the surface. This shows that binding energy of Mn acceptors increases monotonously for decreasing depth below the surface.

The depth dependence of the Mn-induced peaks is reproduced in all other STS measurements even though the absolute peak positions differ. The deviations most probably stem from different tips resulting in modified band bending configurations. All our spectroscopy measurements with sufficient resolution reproduced the threefold splitting of the $\mathrm{Mn}$ induced peak in the band gap. We conclude that all peaks show an enhanced binding energy as predicted by Ref. 15 .

\section{B. Quantitative analysis}

In order to extract the binding energy as a function of the depth of the Mn acceptor below the GaAs(110) surface quantitatively, the TIBB has to be calculated for the voltages at which the Mn-induced features are detected in Fig. 4. As discussed in the previous paragraph and shown in Fig. 5, the Mn peaks in the gap originate from lining up $E_{A}^{\text {surf }}$ with $E_{F}^{\text {bulk }}$. The binding energy is then given by the equation $E_{A}=\left(E_{F}^{b u l k}-E_{V B}\right)+\mid$ TIBB $\mid$. The decay of the TIBB into the material is crucial because the addressed $\mathrm{Mn}$ atoms are located in different depths between $2 \mathrm{AL}$ and $14 \mathrm{AL}$ in the surface. 


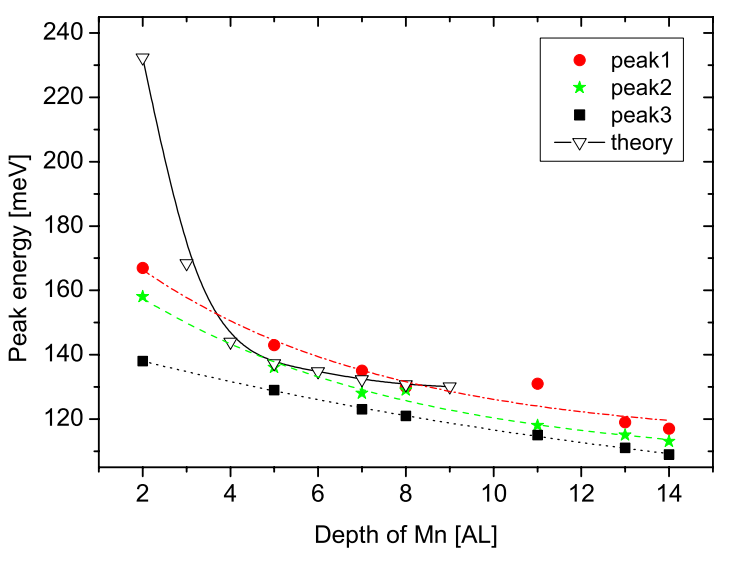

FIG. 6. (Color online) Energetic positions relative to the top of the VB of Mn acceptors in different layers below the $\operatorname{GaAs}(110)$ surface extracted from the experiment. The red dots, green stars, and black squares depict the three peaks in the band gap of GaAs. The open black triangles give the theoretical prediction (Ref. 15).

We calculated the TIBB using the code published in Ref. 26. The obtained binding energy depends on the flat band voltage and on the tip radius $R_{t i p}$. As mentioned before, the flat band voltage was independently determined by $z\left(I_{T}\right)$ spectroscopy to $F B=2.5 \pm 0.5 \mathrm{~V}$. Furthermore we assume similar $R_{t i p}$ as we characterized earlier $R_{t i p} \leq 10 \mathrm{~nm}$ (Refs. 20 and 27) because all our tips are prepared using the same procedure. Although these values seem to have small errors, the resulting variation in $E_{A}$ is still large. Therefore we need further restrictions to narrow down the range of parameters in the TIBB calculations. It is close at hand to assume the binding energy to approach the bulk value asymptotically for acceptors at increasing depth below the surface. Therefore we demand that the calculated binding energies of $\mathrm{Mn}_{13}$ and $\mathrm{Mn}_{14}$ are very similar to each other. $E_{A}^{\mathrm{AL} 13} \approx E_{A}^{\mathrm{AL} 14}$ is achieved for a flat band condition $F B \approx 2.3 \mathrm{~V}$, and a tip radius $R_{t i p}=2 \mathrm{~nm}$, both in good agreement with the expected parameters, $F B=2.5 \pm 0.5 \mathrm{~V}$, and $R_{\text {tip }}<10 \mathrm{~nm}$.

The resulting depth-dependent binding energy is fitted by an exponential decay, depicted by the dashed-dotted red line in Fig. 6. The aymptotic limit of this fit deeply below the surface is $148 \mathrm{meV}, 25 \mathrm{meV}$ higher than the bulk binding energy of $113 \mathrm{meV}$. This deviation most probably stems from a different position of $E_{F}$ in the bulk. We assumed it to be $113 \mathrm{meV}$ above the VB. However, the position of $E_{F}^{b u l k}$ depends on the doping level. Shifting $E_{F}^{\text {bulk }}$ with respect to $E_{V B}$ in the equation we used, $E_{A}=\left(E_{F}^{\text {bulk }}-E_{V B}\right)+\mid$ TIBB $\mid$, results in a constant offset to the extracted binding energies. We removed the offset such that the extracted binding energy asymptotically approaches $113 \mathrm{meV}$ for very deep layers. The results for the investigated $\mathrm{Mn}$ atoms are plotted in Fig. 6 relative to the top of the VB defined as $V B=0 \mathrm{meV}$. The red dots, green stars, and black squares depict the binding energies of the first, second, and third peak. All energetic positions significantly depend on the depth of the addressed $\mathrm{Mn}$ atom below the surface. For Mn atoms closer to the surface, the Mn-induced states in the band-gap shift to higher energy. The extracted binding energy equals $\sim 117 \mathrm{meV}$ for $\mathrm{Mn}_{14}$, which is close to $E_{A}^{\text {bulk }}$, and increases up to $\sim 170 \mathrm{meV}$ for
TABLE II. Splitting between the Mn-induced peaks $\Delta^{i-j}=E_{A}^{i}-E_{A}^{j} .{ }^{*} E_{A}$ of $\mathrm{Mn}_{11}$ is shifted due to the interaction with neighboring acceptors.

\begin{tabular}{lrcccccc}
\hline \hline & $\mathrm{Mn}_{2}$ & $\mathrm{Mn}_{5}$ & $\mathrm{Mn}_{7}$ & $\mathrm{Mn}_{8}$ & $\mathrm{Mn}_{11}$ & $\mathrm{Mn}_{13}$ & $\mathrm{Mn}_{14}$ \\
\hline$\Delta^{1-2}(\mathrm{meV})$ & 9 & 7 & 7 & 1 & $13^{*}$ & 3 & 4 \\
$\Delta^{2-3}(\mathrm{meV})$ & 20 & 7 & 5 & 8 & 3 & 4 & 4 \\
\hline \hline
\end{tabular}

$\mathrm{Mn}_{2}$. The extracted absolute binding energies depend on the choice of the parameters in order to calculate the TIBB. Therefore it is difficult to give the error bars. The error within one choice of parameters is smaller than $\pm 5 \mathrm{meV}$ but there is a possible scaling of $\pm 30 \%$ by choosing different parameters. The binding energies that we extract from the experiment agree satisfyingly well with a recent theoretical study of Mn atoms closely below GaAs(110) ${ }^{15}$ that is plotted as black triangles. Theory predicts a much stronger increase in $E_{A}^{\text {surf }}$ in AL1 and AL2, which is not found in the experiment whereas the furtherly predicted decay toward the bulk value on a larger length scale fits much better with our experimental results.

Now we focus on the Mn-induced feature consisting of three peaks. The Mn $3 d^{5}$ electrons with a total spin of $S=5 / 2$, the hole in the acceptor state with an orbital momentum $L=1$ and a spin of $s=1 / 2$ allow in total 36 angular-momentum states. We define the total momentum $J=S+s+L$, and the 36 states correspond to one $J=1$, two $J=2$, two $J=3$ and one $J=4$ multiplet. The crystal field splits all multiplets with $J>1$, so there are many energetic levels possible. The ground state is given by $J=1$, and if we assume the other two levels visible are two of the excited levels (e.g., $J=2,3$ ), then the splitting between the ground state and the exited states can be assumed to originate either from exchange coupling or from spin-orbit interaction. Exchange coupling would produce progressive splitting between $J=1,2,3$. Table II lists the splitting between the peaks in our experiment for comparison. $\Delta^{i-j}$ denotes the spectroscopic distance between neighboring peaks $i$ and $j$ corresponding to the same Mn atom. Our experimental data clearly do not show progressive splitting so we exclude exchange coupling as the source of the splitting into three levels.

For spin-orbit interaction the states with $J=1,2,3$ are evenly split by $\sim 40 \mathrm{meV}, 25$ which appears superficially similar to the results in Table II. The spin-orbit interaction was also proposed as an explanation for the multiple levels measured in Ref. 21. The calculation of the spin-orbit splitting for Mn in bulk GaAs, ${ }^{25}$ however, has to be compared to the peaks associated with the $\mathrm{Mn}$ atoms in the deepest layers, which are split by 3-4 $\mathrm{meV}$ in the experiment. This is one order of magnitude smaller than predicted in Ref. 25, so we also exclude spin-orbit interaction as the source of the observed splitting.

We conclude that the threefold splitting in our experimental data does not originate from energy splitting between the $J=1$ and other, higher energy angular-momentum states. Instead we propose that the threefold degeneracy of $J=1$ is lifted close to the surface as a result of symmetry lower than the bulk crystal. The symmetry is broken because the relax- 
ation at the $\mathrm{GaAs}(110)$ surface introduces strain into the lattice, and because the voltage applied between the sample and the STM tip results in an electric field at the position of the acceptor. Both effects break the symmetry of acceptor wave functions. ${ }^{12,14,28}$ Under these conditions $m_{J}=+1,0,-1$, are no longer the proper energy eigenstates. However, we assume that three linear combinations will be formed, as described in Refs. 14 and 28, and lead to the threefold splitting of the Mn state in our spectra. Strain or electric fields directed along the [110] direction will produce evenly split states whereas strain or electric fields along the [111] direction will produce two degenerate states and one split-off one. We conclude from Table II that deep within the crystal the strain or electric field is directed along the surface normal, corresponding to evenly split states whereas at the surface there is a strong component of the strain which is along the [111] direction and produces an uneven energy splitting. This effect of strain along the [111] direction is consistent with the results of Ref. 12. Since the states connected to $J=1$ are fully degenerate in the bulk, a small splitting of only $3 \mathrm{meV}$ in our experiment 14 layers deep is not surprising.

\section{CONCLUSIONS}

In summary, the spectroscopic signature of $\mathrm{Mn}$ atoms in different layers below GaAs(110) was measured by scanning tunneling spectroscopy. The observed features, a threefold split peak in the band gap, shift to higher voltage for Mn atoms closer to the surface. The peaks in the band gap are followed by NDC which is explained by an energy-filter model at negative TIBB. This allows the qualitative conclusion that the binding energy of $\mathrm{Mn}$ atoms is enhanced close to the surface. Quantitatively the binding energy was extracted from the measured peak positions by calculating the TIBB in the respective depth below the surface. The resulting binding energy of $\sim 170 \mathrm{meV}$ for $\mathrm{Mn}$ atoms close the GaAs(110) surface is in satisfactory agreement with a recent theoretical prediction. ${ }^{15}$ The threefold splitting of the Mn state is not identified with the theoretical prediction of a threefold state split by the spin-orbit interaction, ${ }^{25}$ or as due to exchange coupling. Instead, we assign it to a splitting of the $J=1$ ground state by the strain and electric field present at the surface.

\section{ACKNOWLEDGMENTS}

We thank M. Bozkurt, C. Çelebi, S. Loth, and M. Wenderoth for valuable discussions, and the STW-VICI under Grant No. 6631, NAMASTE, and COBRA for financial support. *j.k.garleff@tue.nl

${ }^{1}$ J. Bardeen and H. W. Brattain, Phys. Rev. 74, 230 (1948).

${ }^{2}$ S. Roy and A. Asenov, Science 309, 388 (2005).

${ }^{3}$ T. Dietl, F. Matsukura, and H. Ohno, Phys. Rev. B 66, 033203 (2002).

${ }^{4}$ T. Dietl, Semicond. Sci. Technol. 17, 377 (2002).

${ }^{5}$ P. I. Arseev, N. S. Maslova, V. I. Panov, S. V. Savinov, and C. v. Haesendock, JETP Lett. 77, 172 (2003).

${ }^{6}$ A. M. Yakunin, A. Yu. Silov, P. M. Koenraad, J. H. Wolter, W. Van Roy, J. De Boeck, J.-M. Tang, and M. E. Flatté, Phys. Rev. Lett. 92, 216806 (2004).

${ }^{7}$ D. Kitchen, A. Richardella, J.-M. Tang, M. E. Flatté, and A. Yazdani, Nature (London) 442, 436 (2006).

${ }^{8}$ C. Çelebi, P. M. Koenraad, A. Yu. Silov, W. Van Roy, A. M. Monakhov, J.-M. Tang, and M. E. Flatté, Phys. Rev. B 77, 075328 (2008).

${ }^{9}$ J. Tersoff and D. R. Hamann, Phys. Rev. Lett. 50, 1998 (1983).

${ }^{10} \mathrm{~S}$. Loth, M. Wenderoth, and R. G. Ulbrich, Phys. Rev. B 77, 115344 (2008).

${ }^{11}$ J.-M. Jancu, J.-C. Girard, M. O. Nestoklon, A. Lemaître, F. Glas, Z. Z. Wang, and P. Voisin, Phys. Rev. Lett. 101, 196801 (2008).

${ }^{12}$ C. Çelebi, J. K. Garleff, A. Yu. Silov, A. M. Yakunin, P. M. Koenraad, W. Van Roy, J.-M. Tang, and M. E. Flatté, Phys. Rev. Lett. 104, 086404 (2010).

${ }^{13}$ J. K. Garleff, C. Çelebi, W. Van Roy, J.-M. Tang, M. E. Flatté, and P. M. Koenraad, Phys. Rev. B 78, 075313 (2008).

${ }^{14}$ A. M. Yakunin, A. Y. Silov, P. M. Koenraad, J.-M. Tang, M. E. Flatté, J.-L. Primus, W. van Roy, J. de Boeck, A. M. Monakhov, K. S. Romanov, I. E. Panaiotti, and N. S. Averkiev, Nature Mater. 6, 512 (2007).

${ }^{15}$ T. O. Strandberg, C. M. Canali, and A. H. MacDonald, Phys.
Rev. B 80, 024425 (2009).

${ }^{16}$ J. K. Garleff, M. Wenderoth, K. Sauthoff, R. G. Ulbrich, and M. Rohlfing, Phys. Rev. B 70, 245424 (2004).

${ }^{17}$ R. J. Hamers and D. F. Padowitz, in Methods of Tunneling Spectroscopy with the STM in Scanning Tunneling Microscopy and Spectroscopy, Theory, Techniques and Applications, edited by D. Bonnell (VCH, New York, 1993).

${ }^{18}$ F. Marczinowski, J. Wiebe, F. Meier, K. Hashimoto, and R. Wiesendanger, Phys. Rev. B 77, 115318 (2008).

${ }^{19}$ R. M. Feenstra and J. A. Stroscio, J. Vac. Sci. Technol. B 5, 923 (1987).

${ }^{20}$ A. P. Wijnheijmer, J. K. Garleff, K. Teichmann, M. Wenderoth, S. Loth, R. G. Ulbrich, P. A. Maksym, M. Roy, and P. M. Koenraad, Phys. Rev. Lett. 102, 166101 (2009).

${ }^{21}$ F. Marczinowski, J. Wiebe, J.-M. Tang, M. E. Flatté, F. Meier, M. Morgenstern, and R. Wiesendanger, Phys. Rev. Lett. 99, 157202 (2007).

${ }^{22}$ S. Loth, M. Wenderoth, L. Winking, R. G. Ulbrich, S. Malzer, and G. H. Döhler, Phys. Rev. Lett. 96, 066403 (2006).

${ }^{23}$ A. P. Wijnheijmer, J. K. Garleff, M. A. v. d. Heijden, and P. M. Koenraad (unpublished).

${ }^{24}$ A. Richardella, P. Roushan, S. Mack, B. Zhou, D. A. Huse, D. D. Awschalom, and A. Yazdani, Science 327, 665 (2010).

${ }^{25}$ J.-M. Tang and M. E. Flatté, Phys. Rev. Lett. 92, 047201 (2004).

${ }^{26}$ R. M. Feenstra, J. Vac. Sci. Technol. B 21, 2080 (2003).

${ }^{27}$ K. Teichmann, M. Wenderoth, S. Loth, R. G. Ulbrich, J. K. Garleff, A. P. Wijnheijmer, and P. M. Koenraad, Phys. Rev. Lett. 101, 076103 (2008).

${ }^{28}$ J.-M. Tang, J. Levy, and M. E. Flatté, Phys. Rev. Lett. 97, 106803 (2006). 